\title{
High Transmittance In-Plane Switching Liquid Crystal Displays
}

\author{
Zhibing Ge, Student Member, IEEE, Xinyu Zhu, Thomas X. Wu, Senior Member, IEEE, and
} Shin-Tson $\mathrm{Wu}$, Fellow, IEEE

\begin{abstract}
We have developed a high-transmittance in-plane switching (HT-IPS) liquid crystal display (LCD) using special electrode design on the same substrate. The featured electrode configuration generates substantial horizontal electric fields between the electrode groups, and fringe fields with rich horizontal components within the regions of electrode groups. The former is similar to the IPS mode and the latter is similar to the fringe-field switching (FFS) mode. The HT-IPS mode exhibits a high transmittance ( $>90 \%$ of twisted nematic (TN) mode), as well as wide viewing angle for cells using both positive and negative dielectric anisotropy LC materials. In addition, we also compare the electro-optical performance of the HT-IPS with IPS and FFS cells under the same conditions.
\end{abstract}

Index Terms - In-plane switching (IPS), liquid crystal display (LCD), wide-viewing angle.

\section{INTRODUCTION}

$\mathbf{L}$ IQUID CRYSTAL display (LCD) has been extensively employed for notebook computers, desktop monitors, and TV's owing to the continuous improvement in its image quality. Among various LCD technologies, the in-plane switching (IPS) mode [1], [2] is one of the mainstream technologies developed for wide viewing angle. In IPS mode, the inter-digitated electrodes are fabricated on the same substrate and LC molecules are initially homogeneously aligned. The transmission axis of the polarizer can be set to be parallel or perpendicular to the $\mathrm{LC}$ directors while the analyzer is crossed to the polarizer. The in-plane electric fields induced by the electrodes twist the LC directors, thus generate light transmission. However, due to the strong vertical electric field existing above the electrode surface, the LC directors in these regions mainly tilt rather than twist. As a result, the transmittance above the electrodes is greatly reduced. Overall, the conventional IPS mode has a light efficiency about $76 \%$ of that of a twisted nematic (TN) [3] LCD mode, when a positive dielectric anisotropy $(\Delta \varepsilon)$ LC material is used. Although using a negative $\Delta \varepsilon$ liquid crystal in the IPS mode could enhance the light efficiency to above $85 \%$, the drawback is the increased driving voltage.

Manuscript received January 31, 2006; revised March 6, 2006. This work is supported by Toppoly Optoelectronics Corporation (Taiwan), Chu-Nan, Taiwan, R.O.C..

Z. Ge and T. X. Wu are with the School of Electrical Engineering and Computer Science, University of Central Florida, Orlando, FL 32816 USA (e-mail: zge@mail.ucf.edu; tomwu@mail.ucf.edu).

X. Zhu and S. T. Wu are with the College of Optics and Photonics, University of Central Florida, Orlando, FL 32816 USA (e-mail: xzhu@mail.ucf.edu; swu@mail.ucf.edu).

Digital Object Identifier 10.1109/JDT.2006.874502
To enhance the transmittance of the IPS mode, the fringe-field switching (FFS) mode [4]-[6] decreases the electrode width and gap to introduce more horizontal electric fields above and near the electrode surfaces. Consequently, the strong horizontal field is able to twist the LC directors resulting in a high transmittance throughout the whole area. The FFS mode using a negative $\Delta \varepsilon$ material can achieve 98\% transmittance of that of a TN cell. The idea of using a positive $\Delta \varepsilon$ LC material in the FFS mode for achieving high transmittance ( $\sim 90 \%$ of TN mode) is also attempted [6]. It assumes a fine fabrication of electrode width by $1 \mu \mathrm{m}$ that requires a high driving voltage $\left(>6.5 \mathrm{~V}_{\mathrm{rms}}\right)$ in order to generate enough twist to the LC directors. Further optimization for FFS mode using a positive $\Delta \varepsilon \mathrm{LC}$ material is still under active research.

In this paper, we demonstrate a high transmittance in-plane switching (HT-IPS) mode having similar initial LC alignment as the conventional IPS mode, but showing high transmittance ( $>90 \%$ of TN mode) for both positive and negative $\Delta \varepsilon \mathrm{LC}$ materials employed. The electrode design in the HT-IPS mode greatly enhances the transmittance above the electrode surfaces by introducing more horizontal electric fields in these regions, but keeping a similar electric field profile with substantial horizontal components between electrode groups as the conventional IPS mode. With this electrode configuration, the LC directors experience substantial twist throughout the whole area, but the driving voltage required can be reduced to below $6.0 \mathrm{~V}_{\mathrm{rms}}$. Besides, this HT-IPS mode also shows a very wide viewing angle, same as the conventional IPS mode.

\section{Device Configuration And Mechanism}

Fig. 1(a) and (b) shows the typical structures with calculated equal potential lines in the LC cell region for the conventional IPS mode and FFS mode, respectively. In the IPS cell, the electrode width $w$ and separation distance $l$ are usually kept larger than the cell gap $d$. From Fig. 1(a), we can see that substantial horizontal electric fields exist only between the electrodes, and both vertical and horizontal electric fields exist above the electrode surface. Furthermore, the horizontal electric fields above the electrode surface are weaker than the vertical ones, which tilt the LC directors there and cause a low transmittance in IPS mode. As for the FFS cell in Fig. 1(b), both the electrode width $w$ and the horizontal distance between the pixel electrode and the common electrode (zero in this case) are kept smaller than the cell gap $d$. In such a configuration, horizontal electric fields peak at the edges of electrodes and decrease to the minimum in the symmetric centers above the electrode and above the slot. Compared to the IPS mode, the horizontal electric fields at the electrode edges in the FFS cell are stronger, since the potential 

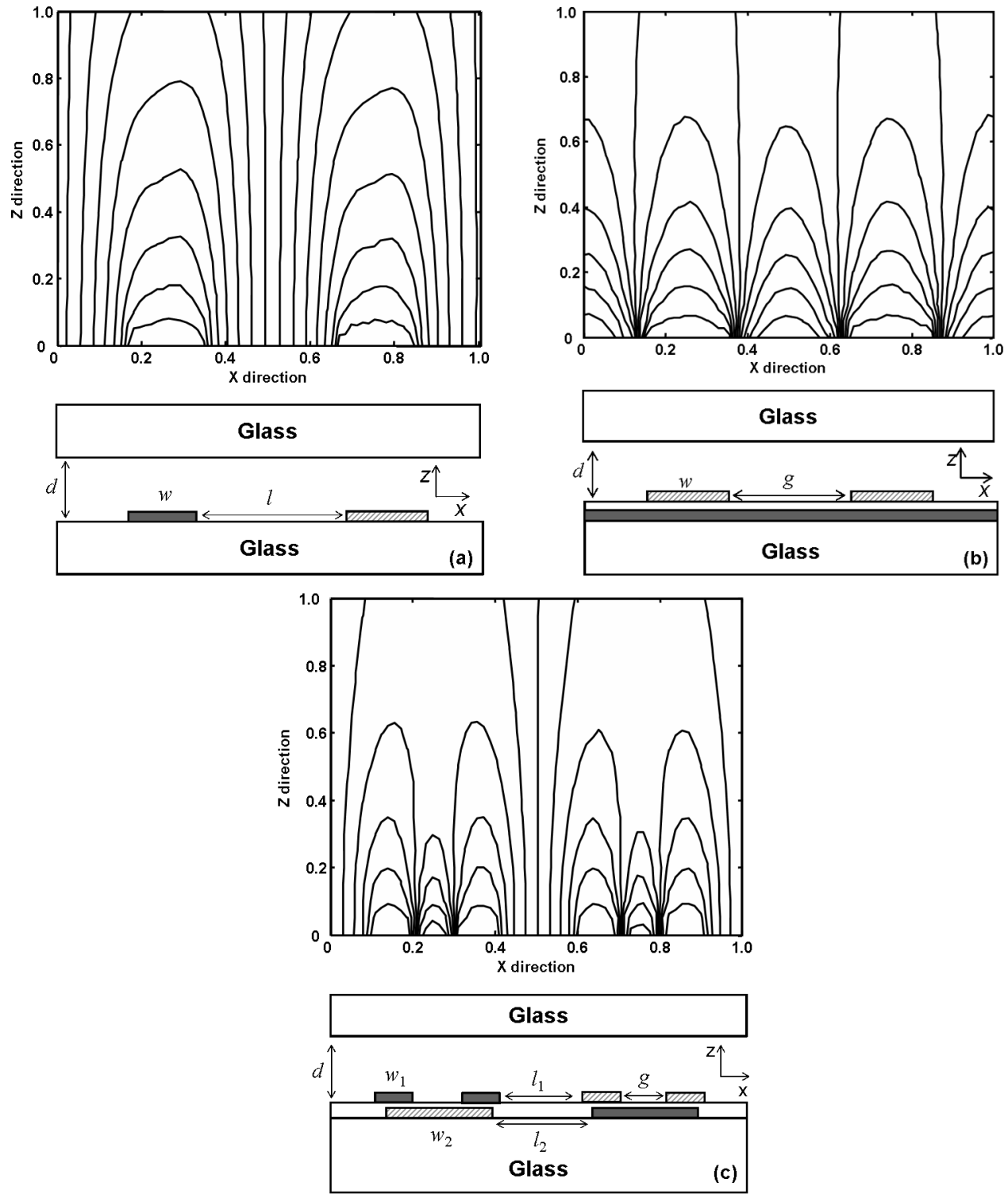

Fig. 1. Structure and potential profile of: (a) an IPS cell; (b) an FFS cell; and (c) an HT-IPS cell.

lines there are denser. Therefore, once the director tilt is suppressed by using a negative $\Delta \varepsilon$ LC, strong horizontal electric fields will first twist the LC directors at these edges, and further rouse the twist of the other directors in the centers of the electrode surface and the slot. However, because of the existence of both horizontal and vertical electric field components above these symmetric centers, the positive $\Delta \varepsilon$ LC directors still experience tilt. As the directors start to tilt, the effective twist torque projected in the plane will be reduced. Consequently, this edge-to-center process requires a much stronger electric field than the case using a negative $\Delta \varepsilon$ LC.

Fig. 1(c) shows a design of the HT-IPS mode with potential lines included. The motivation is to keep a similar substantial horizontal field region like IPS mode, but introduce fringe fields with more horizontal electric field components only in the regions above electrodes. In Fig. 1(c), the driving electrodes are formed on two different planes isolated by a passivation layer. Each pixel electrode in the bottom layer is adjoined by two common electrodes on the upper layer, which together form an electrode group showing a potential similar to a common electrode in the IPS mode. Correspondingly, each common electrode in the bottom layer adjoined with two pixel electrodes from the upper layer work as an electrode group like a pixel electrode in the IPS mode. Besides, within each subgroup, the electrode adjoining the LC in the upper layer has a width $w_{1}$ smaller than the cell gap $d$, and the horizontal distance between the common and pixel electrodes [zero in the design shown in Fig. 1(c)] is smaller than the cell gap $d$ as well. Therefore, much richer horizontal electric fields combined with the vertical components are generated within each electrode group than they are in the conventional IPS cell. However, to maintain the substantial horizontal electric fields generated between different electrode groups as in the IPS mode, the distance between adjacent electrodes from two electrode groups, such the distance $l_{1}$ and $l_{2}$ is kept larger than the cell gap $d$. The characteristics of this desired electric field distribution can clearly be verified by the calculated potential profile shown in Fig. 1(c). Consequently, it can achieve a higher transmittance than the IPS mode and lower 

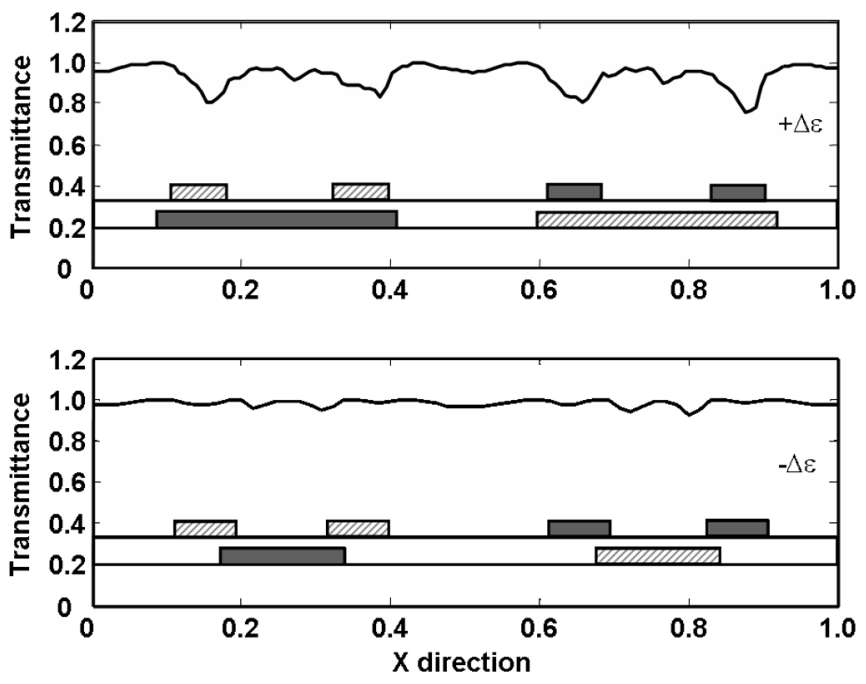

Fig. 2. Transmittance curves for HT-IPS cells with $+\Delta \varepsilon$ and $-\Delta \varepsilon$ LC's.

driving voltage than the FFS mode using a positive $\Delta \varepsilon$ LC material, but its electrode configuration is somewhat more complicated than the IPS and FFS modes.

\section{RESULTS AND DISCUSSION}

We have investigated the HT-IPS mode with both positive ( $\Delta \mathrm{n}=0.095$ at $\lambda=589 \mathrm{~nm}$, and $\Delta \varepsilon=10.0)$ and negative $(\Delta \mathrm{n}=0.083$ at $\lambda=589 \mathrm{~nm}, \Delta \varepsilon=-4.2) \Delta \varepsilon$ LC materials. The cell parameters are listed in Table I. The electro-optical performance is calculated by the commercial LCD simulator 2dimMOS [7] and the extended Jones matrix method [8].

Fig. 2 shows the transmission with respect to the position in a single electrode period for HT-IPS cells using positive and negative $\Delta \varepsilon$ LC's. The corresponding electrode position is also included in the figure as a reference. The averaged light efficiency for cells using positive and negative $\Delta \varepsilon$ materials is around $93 \%$ and $98 \%$ of that of a TN cell in accordance with the top and bottom figures. The driving voltage of a thin-film transistor (TFT) is preferably around $5.5 \mathrm{~V}_{\mathrm{rms}}$. For the cell using a negative $\Delta \varepsilon$ material, the transmittance is almost flat, as the tilt is suppressed there. The transmittance in the positive $\Delta \varepsilon$ LC cell is nearly flat only in the regions between electrode groups where horizontal fields dominate, and within each electrode group, fringe fields that have rich horizontal components twist the LC directors and give rise to a high transmittance as well. From both curves, there are still some low transmittance kinks. A detailed view shows these kinks occur above the electrode surfaces in the cell using a positive $\Delta \varepsilon$ material, but they appear near the inner edges of upper electrodes within each group in the negative $\Delta \varepsilon \mathrm{LC}$ cell.

The mechanism responsible for these kinks in two cells is different. In the upper curve using a positive $\Delta \varepsilon$ material, the LC directors interact with the strong vertical electric fields above the electrode surfaces and tilt to cause a relatively low transmittance kink. In the negative $\Delta \varepsilon$ LC cell, no such tilt effect takes place. But because the potential lines are the densest at the inner edges of upper electrodes in each electrode group as shown in Fig. 1(c), the horizontal field almost peaks at those positions. Thus the twist of LC directors there is stronger than it is at other positions. Since the cell parameters are optimized for the overall region, the LC
TABLE I

PARAMETERS USED IN THE HT-IPS CELLS

\begin{tabular}{ccc}
\hline & $+\Delta \varepsilon \mathrm{LC}$ & $-\Delta \varepsilon \mathrm{LC}$ \\
\hline$d$ & $4.0 \mu \mathrm{m}$ & $4.4 \mu \mathrm{m}$ \\
rubbing angle & $80^{\circ}$ & $10^{\circ}$ \\
pretilt angle & $2^{\circ}$ & $2^{\circ}$ \\
$w_{1}$ & $2 \mu \mathrm{m}$ & $2 \mu \mathrm{m}$ \\
$w_{2}$ & $9 \mu \mathrm{m}$ & $4 \mu \mathrm{m}$ \\
$g$ & $4 \mu \mathrm{m}$ & $3 \mu \mathrm{m}$ \\
$l_{1}$ & $6 \mu \mathrm{m}$ & $5 \mu \mathrm{m}$ \\
$l_{2}$ & $5 \mu \mathrm{m}$ & $8 \mu \mathrm{m}$ \\
\hline
\end{tabular}

directors near the inner electrode edges are over-twisted resulting in a relative low transmittance there.

We further study the electro-optical performance such as voltage dependent transmittance (V-T) curves and iso-luminance plots among the IPS, FFS, and HT-IPS modes, because they have the same initial homogeneous alignment and the electrodes are formed on one substrate. The $\mathrm{d} \Delta \mathrm{n}$ value for all the cells using a positive $\Delta \varepsilon \mathrm{LC}$ is kept at $0.38 \mu \mathrm{m}$, and 0.36 $\mu \mathrm{m}$ for those using a negative $\Delta \varepsilon$ material. The wavelength used in simulations is $\lambda=550 \mathrm{~nm}$. The electrode width $w$ in the IPS mode in Fig. 1(a) is $4 \mu \mathrm{m}$ with a separation length $l$ of $8 \mu \mathrm{m}$ for both cells using positive and negative $\Delta \varepsilon$ materials. For the FFS structure in Fig. 1(b), the electrode width $w$ is 2 $\mu \mathrm{m}$ with a separation gap $g$ of $3 \mu \mathrm{m}$ when using a positive $\Delta \varepsilon$ material; and $w$ is $3 \mu \mathrm{m}$ and $g$ is $4.5 \mu \mathrm{m}$ for the cell using a negative $\Delta \varepsilon$ LC. The electrode dimensions for the HT-IPS designs are the same as those listed in Table I.

Fig. 3(a) and (b) compares the simulated V-T curves for cells using both positive and negative $\Delta \varepsilon$ LCs in the IPS, FFS, and HT-IPS modes, respectively. The transmittance is normalized to the maximum light efficiency of a TN cell. The cells using a negative $\Delta \varepsilon$ material show a higher transmittance due to the less tilt there. For the cells using a positive $\Delta \varepsilon$ material, the IPS mode has a maximum transmittance of $\sim 76 \%$ because of the insufficient twist above the electrode surface, while the FFS cell and the HT-IPS cell show a similar maximum transmittance of $\sim 93 \%$. Furthermore, because of its largest distance $l$ between the common electrode and the pixel electrode, the IPS cell in Fig. 3(a) have the highest threshold voltage $\left(1.5 \mathrm{~V}_{\mathrm{rms}}\right)$ as compared to those of the FFS and HT-IPS cells $\left(\mathrm{V}_{\mathrm{th}} \sim 1.0 \mathrm{~V}_{\mathrm{rms}}\right)$. However, its transmittance starts to saturate at the lowest voltage of $4.5 \mathrm{~V}_{\mathrm{rms}}$, while the FFS cell and the HT-IPS cell saturate at $7.0 \mathrm{~V}_{\mathrm{rms}}$ and 5.5 $\mathrm{V}_{\mathrm{rms}}$, respectively. The source of transmittance change in each cell might contribute to this difference in their on-state driving voltages. In the IPS cell, the LC directors above the electrode surfaces mainly tilt. Once they tilt up, their contributions to the overall transmittance change become less important as the voltage increases. In other words, the twist level of the LC directors between the electrodes, where they can be easily rotated, determines the overall transmittance change and the saturation voltage in the IPS cell. However, in the FFS cell, the LC directors throughout the whole cell contribute to the overall light efficiency. And the 

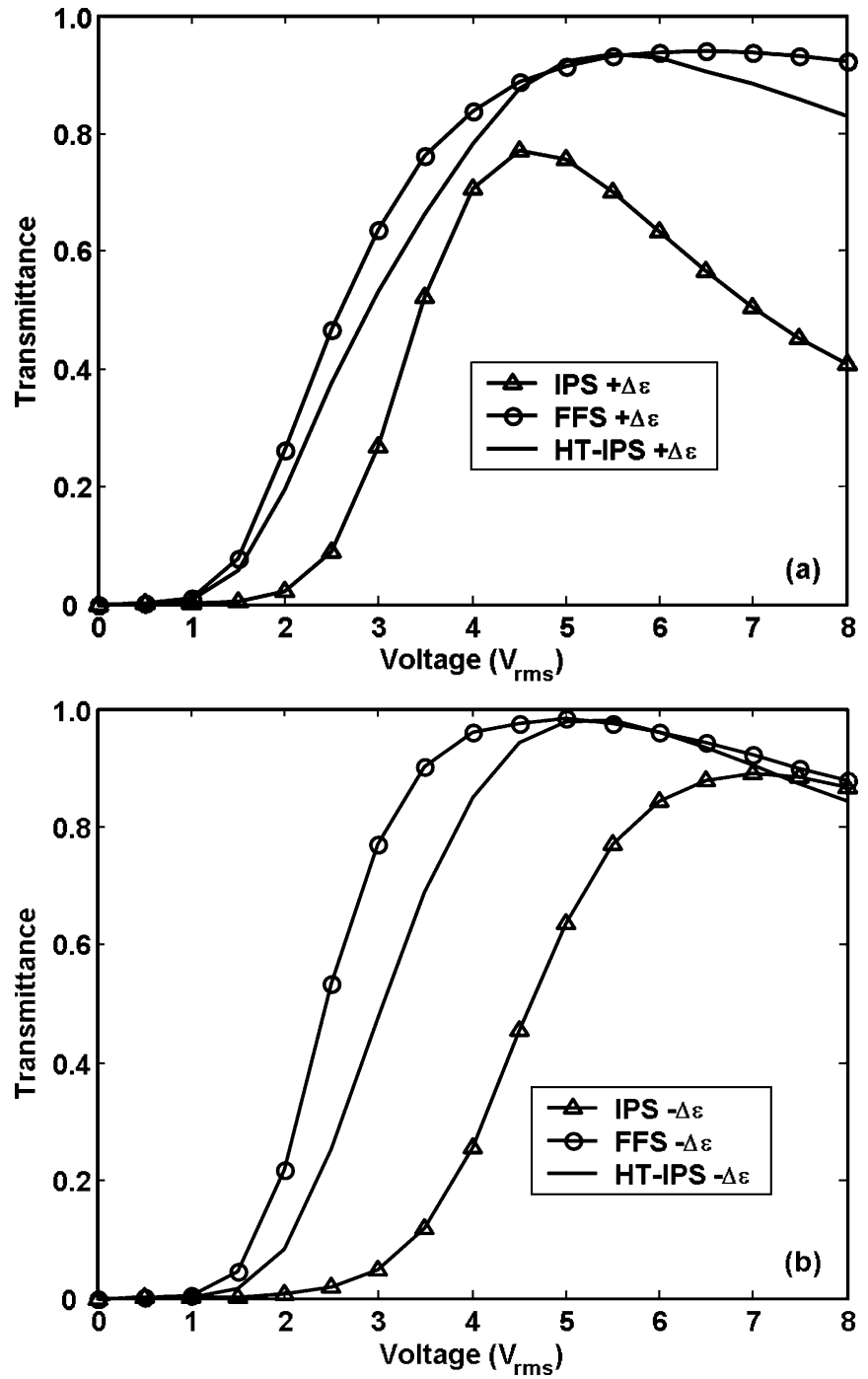

Fig. 3. V-T curves for IPS, FFS and HT-IPS cells using a (a) $+\Delta \varepsilon$ and (b) $-\Delta \varepsilon \mathrm{LC}$.

directors near the electrode edges, where the horizontal electric fields dominate, will twist first at a low voltage. But it needs a high voltage to further rotate those directors near the symmetric centers of the electrode surface and the slot, because the directors there also experience some tilt. As the intermediate state between the pure IPS mode and the FFS mode, the HT-IPS cell shows a moderate driving voltage.

For these cells using a negative $\Delta \varepsilon$ LC in Fig. 3(b), the IPS cell shows a maximum transmittance of $88 \%$, while values in the FFS cell and the HT-IPS cell are both around 98\%. Owing to its cell dimension, the IPS has the highest threshold voltage close to $2.0 \mathrm{~V}_{\mathrm{rms}}$, while the FFS cell and the HT-IPS cell have a similar $\mathrm{V}_{\mathrm{th}} \sim 1.0 \mathrm{~V}_{\mathrm{rms}}$. However, the trend of the on-state driving voltage for cells using different $\Delta \varepsilon$ materials is quite the opposite. The IPS cell here has the highest driving voltage of $7.0 \mathrm{~V}_{\mathrm{rms}}$. The saturation voltages in the FFS cell and the HT-IPS cell are around 5.0 $\mathrm{V}_{\mathrm{rms}}$ and $5.5 \mathrm{~V}_{\mathrm{rms}}$, respectively. Because of the tilt in these negative $\Delta \varepsilon$ LC cells is suppressed, the twist level of the LC directors above the electrode surface will also contribute to the overall transmittance until it reaches a saturation level. As the IPS cell has the weakest horizontal field

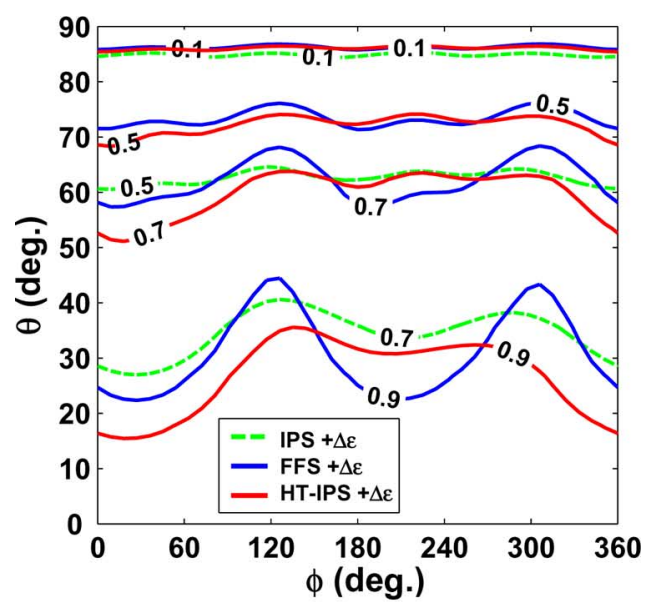

(a)

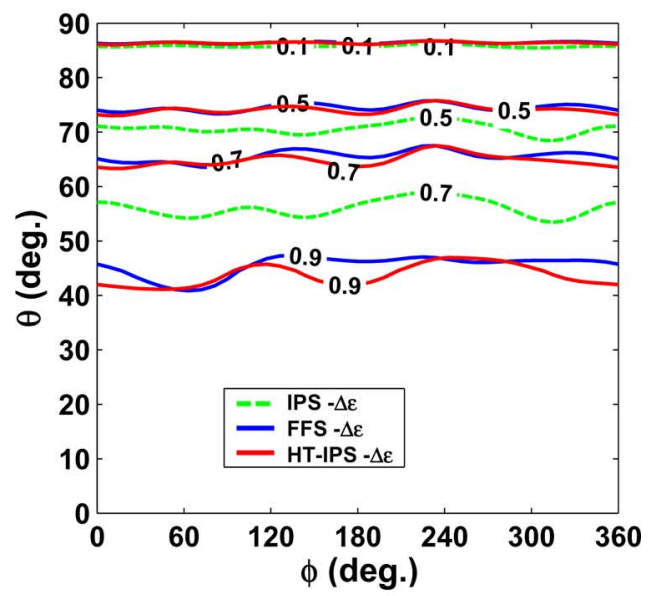

(b)

Fig. 4. Iso-luminance plots for IPS, FFS and HT-IPS cells using a (a) $+\Delta \varepsilon$ and (b) $-\Delta \varepsilon$ LC. (Color version available online at http://ieeexplore.ieee.org.)

components above electrodes, the twist of LC directors there requires a strong driving voltage. Similarly, because the HT-IPS cell fuses the IPS and FFS structures, its saturation voltage has an intermediate value. More detailed reasons to cause the saturation voltage difference should be based on the analysis of their specific on-state configurations.

Besides the electro-optic performance in the normal direction, the on-state iso-luminance at $\mathrm{T}=0.9,0.7,0.5$, and 0.1 for the IPS, FFS, and HT-IPS cells using positive and negative $\Delta \varepsilon$ LC's are further investigated in Fig. 4(a) and (b), respectively. The angle $\theta$ in the figure is defined as the angle between the incident direction and the normal $z$-axis, and $\phi$ is the azimuthal angle away from the $x$-axis in the $x-y$ plane. For those cells using a positive material in Fig. 4(a), the IPS mode is inferior to the FFS and HT-IPS modes in the iso-luminance performance. For the IPS cell, the $\mathrm{T} \geq 70 \%$ region is confined within a $40^{\circ}$ viewing cone at most azimuthal directions, while it is expanded to above $50^{\circ}$ at all directions for the FFS and HT-IPS cells. Furthermore, the FFS cell has its $\mathrm{T} \geq 90 \%$ cone fluctuated between $20^{\circ}$ and $45^{\circ}$. In contrast, the $\mathrm{T} \geq 90 \%$ cone for the HT-IPS cell is ranged between $15^{\circ}$ and $35^{\circ}$.

When a negative $\Delta \varepsilon$ LC is employed, their corresponding iso-luminance cones as shown in Fig. 4(b) are greatly enhanced 

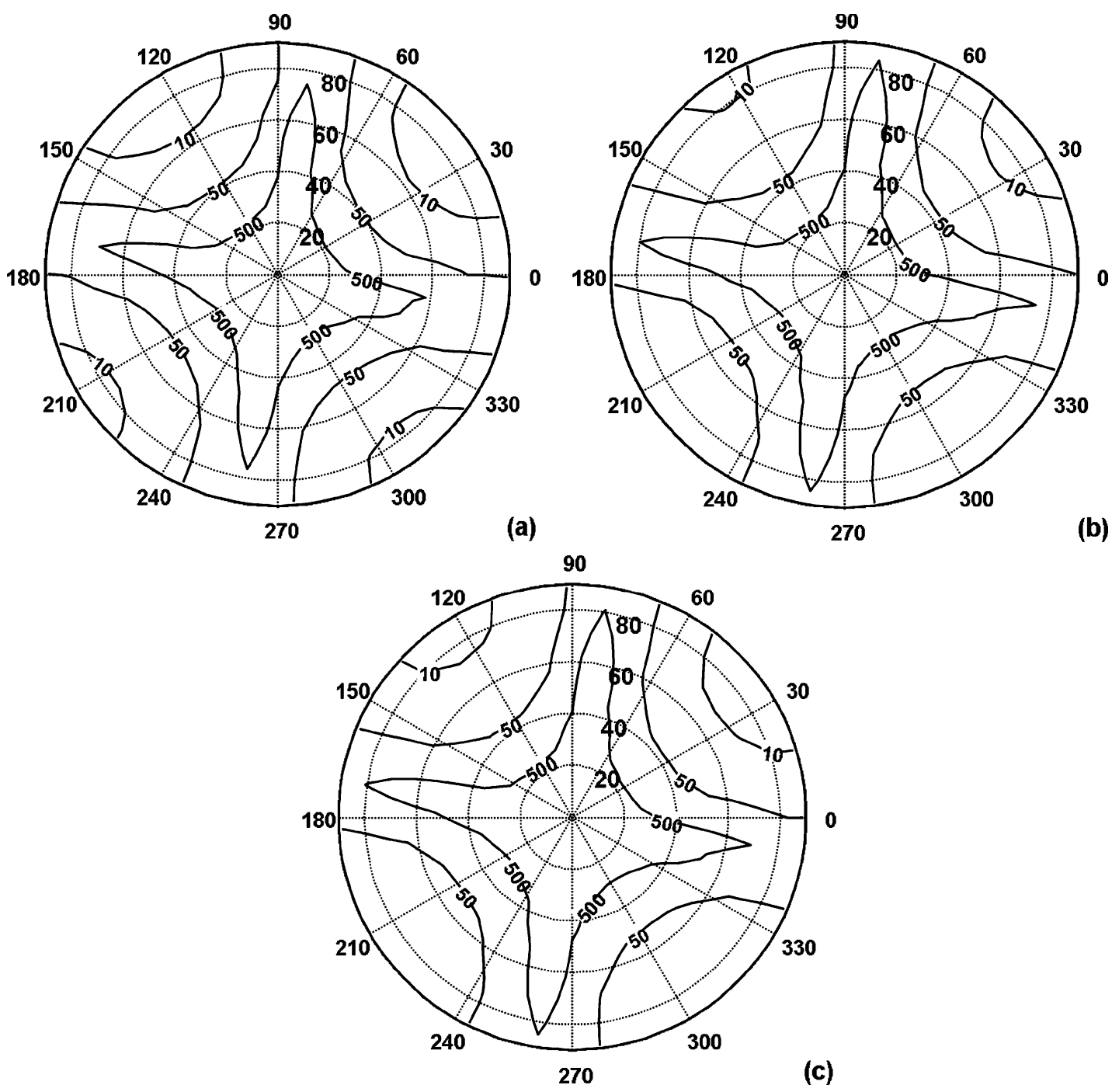

(b)

(c)

Fig. 5. Iso-contrast plots for: (a) an IPS cell; (b) an FFS cell; and (c) an HT-IPS cell using a $+\Delta \varepsilon$ LC.

due to the suppression of tilt. For the IPS cell shown in Fig. 4(b), $\mathrm{T} \geq 70 \%$ is enlarged to a viewing cone above $55^{\circ}$, but no $\mathrm{T} \geq$ $90 \%$ cone exists in the plot. The FFS and HT-IPS cells show a close iso-luminance plot, in which the $\mathrm{T} \geq 90 \%$ and $\mathrm{T} \geq$ $70 \%$ cones are enlarged to above $40^{\circ}$ and $65^{\circ}$, respectively. In addition, because the director distribution in the negative $\Delta \varepsilon$ cells is more uniform, the iso-luminance here is less azimuthal angle dependent than that in the positive $\Delta \varepsilon$ LC cells.

The viewing angle is also investigated for the IPS, FFS, and HT-IPS structures using both positive and negative $\Delta \varepsilon$ LC materials. Fig. 5(a)-(c) shows the iso-contrast plots for the IPS, FFS, and HT-IPS cells using a positive $\Delta \varepsilon$ LC, and Fig. 6(a)-(c) is the corresponding plots for the negative $\Delta \varepsilon$ LC. No compensation film is employed in the calculations. For the cells using a positive (negative) $\Delta \varepsilon$ LC, because the initial LC cells have the same configuration in the dark state, their dark states are identical. Therefore, their bright states will determine the iso-contrast performance difference. As shown in Fig. 4(a) and (b), the FFS and HT-IPS cells have a wider iso-luminance viewing cone than the IPS cell. As a result, their viewing angle is wider as shown in the iso-contrast plots.
For TV applications, wide viewing angle in LCD is critical. Various film compensation schemes of IPS and FFS modes have been developed [9], [10]. The film compensation of the HT-IPS should be similar to those of IPS and FFS modes. In addition to the electrode configuration shown in Fig. 1(c), other similar electrode designs to fuse the IPS and FFS modes are also proposed to improve the transmittance of the conventional IPS mode [11].

\section{CONCLUSION}

In this paper, we demonstrated an HT-IPS LCD with a special electrode configuration to achieve high transmittance, where the electrodes generate substantial horizontal electric fields between electrode groups and fringe fields with rich horizontal components within electrode groups to twist the LC directors throughout the whole cell. Detailed mechanism underneath to attain such high transmittance using both positive and negative $\Delta \varepsilon$ materials is discussed. And the electro-optic performance such as voltage-transmittance curve and iso-luminance plot for the IPS, FFS, and HT-IPS modes is also studied. The detailed origins causing the performance difference among 

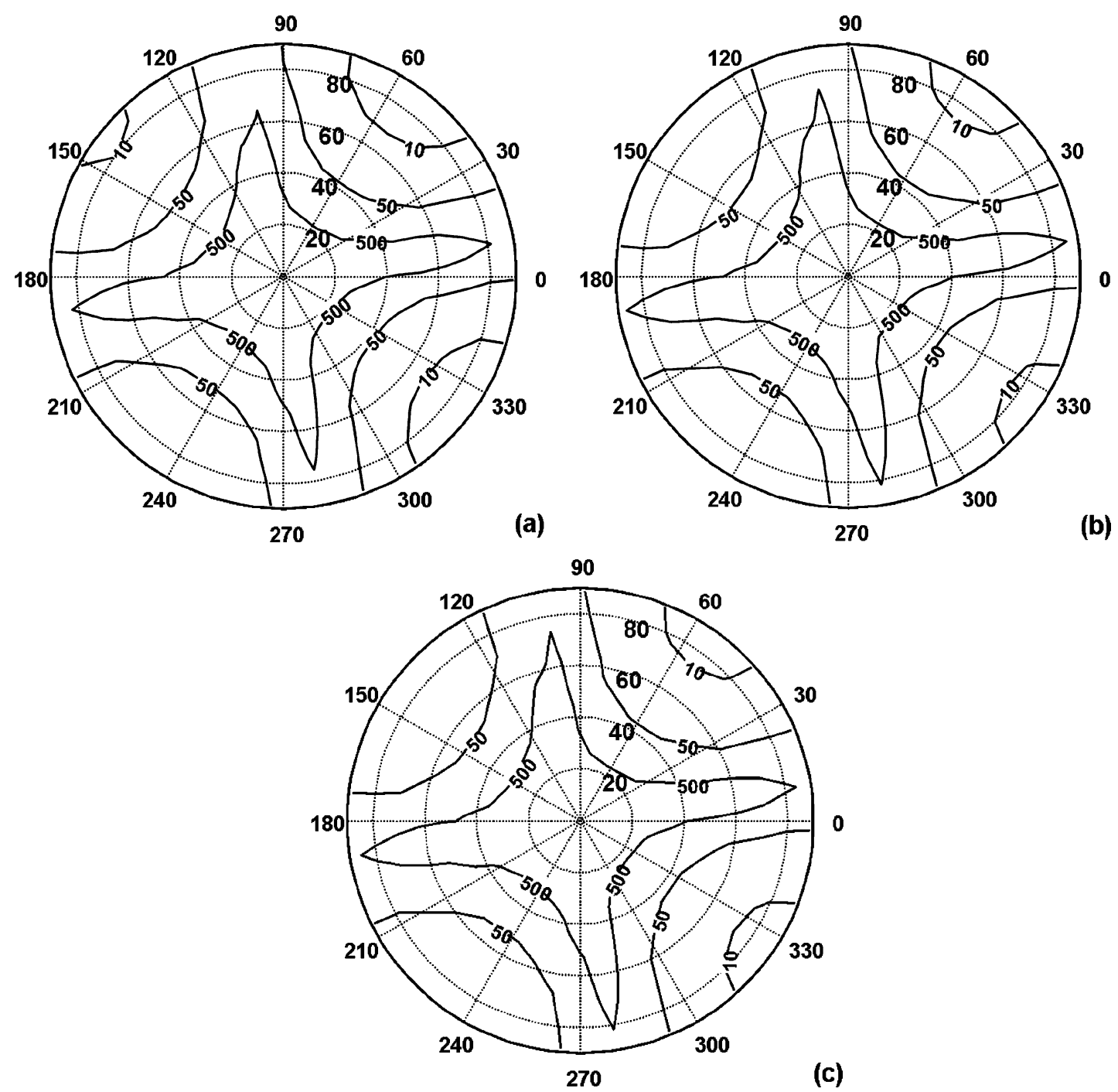

Fig. 6. Iso-contrast plots for: (a) an IPS cell; (b) an FFS cell; and (c) an HT-IPS cell using a $-\Delta \varepsilon$ LC.

different modes are investigated. Besides, the HT-IPS mode shows a very wide viewing even without any compensation films, because of the in-plane rotation of LC directors. The high transmittance IPS mode can be applied to LCD's requiring high transmittance and wide-viewing angle.

\section{REFERENCES}

[1] R. A. Soref, "Field effects in nematic liquid crystals obtained with interdigital electrodes," J. Appl. Phys., vol. 45, pp. 5466-5468, Dec. 1974.

[2] M. Ohe and K. Kondo, "Electro-optical characteristics and switching behavior of the in-plane switching mode," Appl. Phys. Lett., vol. 67, pp. 3895-3897, Oct. 1995.

[3] M. Schadt and W. Helfrich, "Voltage-dependent optical activity of a twisted nematic liquid crystal," Appl. Phys. Lett., vol. 18, pp. 127-128, Feb. 1971.

[4] S. H. Lee, S. L. Lee, and H. Y. Kim, "Electro-optic characteristics and switching principle of a nematic liquid crystal cell controlled by fringefield switching," Appl. Phys. Lett., vol. 73, pp. 2881-2883, Nov. 1998.

[5] S. H. Lee, S. M. Lee, H. Y. Kim, J. M. Kim, S. H. Hong, Y. H. Jeong, C. H. Park, Y. J. Choi, J. Y. Lee, J. W. Koh, and H. S. Park, "18.1" Ultra-FFS TFT-LCD with super image quality and fast response time," in SID Symp. Dig., May 2001, pp. 485-487.

[6] Y. M. Jeon, I. S. Song, S. H. Lee, H. Y. Kim, S. Y. Kim, and Y. J. Lim, "Optimized electrode design to improve transmittance in the fringefield switching (FFS) liquid crystal cell," in SID Symp. Dig., May 2005, pp. 328-331.
[7] Autronic-MELCHERS GmbH [Online]. Available: http://www. autronic-melchers.com/

[8] A. Lien, "Extended Jones matrix representation for the twisted nematic liquid-crystal display at oblique incidence," Appl. Phys. Lett., vol. 57, pp. 2767-2769, Dec. 1990

[9] R. Lu, X. Zhu, S. T. Wu, Q. Hong, and T. X. Wu, "Ultra wide view liquid crystal displays," J. Display Technology, vol. 1, pp. 3-14, Sep. 2005.

[10] X. Zhu, Z. Ge, and S. T. Wu, "Analytical solutions for uniaxial-filmcompensated wide-view liquid crystal displays," J. Display Technol., vol. 2, no. 1, pp. 2-20, Mar. 2006.

[11] Z. Ge, X. Zhu, S. T. Wu, and T. X. Wu, "High transmittance and wide viewing angle liquid crystal display devices,", 2005, U.S. Patent Appl. pending.
Zhibing Ge (S'02) received the B.S. and M.S. degrees in Electrical Engineering from Zhejiang University, Hangzhou, China and University of Central Florida (UCF), Orlando, in 2002 and 2004, respectively, and is currently working toward the Ph.D. degree at the School of Electrical Engineering and Computer Science, UCF, Orlando. His Ph.D. study focuses on transflective liquid crystal displays. 
Xinyu Zhu received the Ph.D degree from Changchun Institute of Optics, Fine Mechanics and Physics, Chinese Academy of Sciences, China in 2001 and B.S degree from Jilin University, China in 1996. He joined the College of Optics and Photonics, UCF as a research scientist in 2001.

His current research interests include reflective and transflective liquid crystal displays, liquid crystal on silicon ( $\mathrm{LCOS}$ ) projection display, wide viewing angle liquid crystal displays and adaptive optics application with nematic liquid crystals.

Thomas X. Wu received the B.S.E.E. and M.S.E.E. degrees from the University of Science and Technology of China (USTC), Anhui, China, in 1988 and 1991, respectively, and the M.S. and Ph.D. degrees in electrical engineering from the University of Pennsylvania, Philadelphia, in 1997 and 1999, respectively.

$\mathrm{He}$ is currently an associate professor at the School of Electrical Engineering and Computer Science, UCF, Orlando. His current research interests and projects include complex media, liquid crystal devices, electronic packaging of RF SAW devices, electrical machinery, magnetics and EMC/EMI in power electronics, chaotic electromagnetics, millimeter-wave circuits, and CMOS/BiCMOS RFIC's.

Dr. Wu received the Distinguished Researcher Award from the College of Engineering and Computer Science, UCF, in April 2004. He is listed in Who's Who in Science and Engineering, Who's Who in America, and Who's Who in the World.
Shin-Tson Wu (M'98-SM'99-F'04) received the B.S. degree in physics from National Taiwan University, Taipei, and the Ph.D. degree from the University of Southern California, Los Angeles.

$\mathrm{He}$ is currently a PREP professor at College of Optics and Photonics, University of Central Florida, Orlando. His studies at UCF concentrate in foveated imaging, bio-photonics, optical communications, liquid crystal displays, and liquid crystal materials. He has coauthored 3 books, Fundamentals of Liquid Crystal Devices (Wiley, 2006), Reflective Liquid Crystal Displays (Wiley, 2001) and Optics and Nonlinear Optics of Liquid Crystals (World Scientific, 1993), 4 book chapters, over 300 papers, and 55 issued and pending patents.

Dr. Wu is a Fellow of the Society for Information Display (SID) and Optical Society of America (OSA). 